\title{
Avances tecnológicos en gestión de vertimientos líquidos en plantas de producción de alimentos
}

\section{Technological advances in liquid watershed management from food production centers}

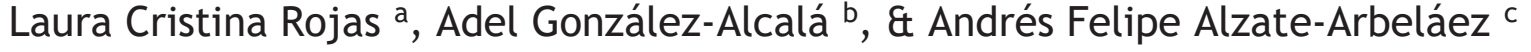 \\ a Gestor de Proyectos, Red Tecnoparque SENA Nodo Medellín - Grupo GIGAT, Servicio Nacional de Aprendizaje SENA, \\ Medellín-Colombia Icrojasb@sena.edu.co \\ ${ }^{b}$ Coordinador del Programa Gestión del Emprendimiento y la Innovación - Universidad Pontificia Bolivariana, Medellín- \\ Colombia, adel.gonzalez@upb.edu.co \\ ${ }^{c}$ Escuela de Química, Facultad de Ciencias, Universidad Nacional de Colombia-Sede Medellín, Colombia, \\ afalzatea@unal.edu.co
}

Recibido: agosto 30, 2019. Aceptado: noviembre 19, 2019

\begin{abstract}
Resumen
En la presente revisión se hace una búsqueda, análisis y recopilación de información de carácter científico, tecnológico y comercial en el tema de vertimientos líquidos en industrias de alimentos. Se definieron palabras claves y restricciones pertinentes, se revisaron alrededor de 40 artículos científicos y 20 patentes que cumplían con los criterios de búsqueda. Los resultados indican que el proceso más usado para el tratamiento de residuos líquidos es mediante su deshidratación y tratamiento en plantas de aguas residuales; sin embargo, debido a su composición, es relevante implementar economía circular y usar dichos subproductos como materia prima para la producción de jarabes con alto contenido de azúcar, producción de alcoholes, enzimas, biopolímeros, entre otros. El análisis cienciométrico indica que España y Estados Unidos son pioneros en las investigaciones y patentes en el tema, mientras Colombia no presenta publicaciones entre 2008 y 2018.

Palabras clave: Industria De Alimentos, Vertimientos, Gestión de vertimientos, residuos líquidos.
\end{abstract}

\section{Abstract}

In this review, a data search and analysis are performed on the issue of liquid discharges in food industries. Information was collected from scientific, technological, and commercial nature. Relevant keywords and restrictions were defined, and about 40 scientific articles and 20 patents that met the search criteria were reviewed. The results indicate that the most used process for the treatment of liquid waste relies on dehydration and processing in wastewater plants. However, due to its composition, it is essential to implement a circular economy, and use the produced by-products as raw material to produce syrups with high sugar content, production of alcohols, enzymes, biopolymers, among others. The Scientometric analysis indicates that Spain and the United States are pioneers in research and patents on the subject, while Colombia does not present publications between 2008 and 2018.

Keywords: Food industry, liquid waste, liquid waste management.

\section{INTRODUCCIÓN}

En un mundo moderno, sobrepoblado y en constante desarrollo, donde se busca conservar los recursos naturales a las futuras generaciones, la producción de residuos o subproductos es un tema álgido en términos ambientales. Con el fin de crear un mecanismo para un correcto manejo de los subproductos se creó la tabla de jerarquía de manejo de estos [1], donde se prioriza en términos de subproductos la prevención en la generación, posteriormente en la escala (según nivel de importancia) le siguen su reducción, reutilización y reciclaje, siendo la última opción la disposición la cual se espera que afecte lo menos posible el medio ambiente y, en general, los recursos naturales.

En el sector alimenticio, un residuo es definido como cualquier subproducto generado en la cadena de suministro del alimento, bien sea en su producción, venta o consumo; estos residuos no son reciclados o utilizados de manera definitiva, pudiendo generar problemas medioambientales (malos olores y putrefacción) y siendo un foco de enfermedades bajo un mal manejo [2][3]. Un estudio publicado por la Unión Europea en el año 2010 indica que aproximadamente 90 millones de toneladas de residuos alimenticios son generados a nivel industrial

\footnotetext{
Citar como:

L. Rojas, A. González, A. Alzate-Arbeláez. "Avances tecnológicos en gestión de vertimientos líquidos en plantas de producción de alimentos" Revista CINTEX, Vol. 24(1), pp. 21-32. 2019.
} 
cada año [4]. Hablando específicamente de los residuos líquidos, tales efluentes tienen gran cantidad de sólidos suspendidos, nitrógeno, grasas, aceites y en general material orgánico [5].

Para un correcto tratamiento de los subproductos generados, las empresas deben asumir costos de vertimiento y tratamiento para cumplir con la normativa asociada al tema; tales costos dependen en gran parte de la naturaleza, características y grado de contaminación generada por los subproductos. Sin embargo, en los últimos años, la creciente preocupación por el medio ambiente ha llevado a las autoridades y las empresas a buscar soluciones económicamente viables para el reciclaje o valorización de productos de desecho, logrando así evitar o reducir los procesos de disposición final [6].

Existen reportes donde se ha mencionado de los subproductos alimenticios podrían pasar de ser un desperdicio a un tesoro, lo anterior teniendo en cuenta la composición y las cantidades generadas de subproductos en industrias de alimentos; así entonces, la idea de convertir tal material en energía o materia prima de gran interés a nivel industrial hacen de su aprovechamiento un área emergente, con gran potencial y oportunidades [7]. Dados estos antecedentes, en el presente trabajo se presentan los aspectos más relevantes del análisis de información científica, tecnológica y comercial disponible acerca del tratamiento de vertimientos líquidos que genera la industria de alimentos y las alternativas de aprovechamiento.

\section{MATERIALES Y MÉTODOS}

Para el desarrollo de la vigilancia tecnológica se tuvo en cuenta la metodología InnoViTech (Innovaciones a partir de la Vigilancia tecnológica). Ésta es una metodología flexible que consiste en el diseño y ejecución compartida de vigilancias tecnológicas para reforzar la toma de decisiones estratégicas a empresas, emprendedores y grupos de investigación. El proceso se desarrolla en función de la toma de decisiones, apoyado con el desarrollo de unas fases cíclicas: identificación de las necesidades, diagnóstico, definición del factor crítico, búsqueda y recolección de información, análisis, elaboración de informes y difusión del modelo. Tales fases permiten la retroalimentación en cualquier momento, de acuerdo con los resultados obtenidos en cada etapa [8].

\subsection{Planeación de la vigilancia tecnológica}

Se eligió un intervalo de 8 años para la búsqueda y análisis de información, adicionalmente el factor crítico de la vigilancia elegido fue: identificación de la información científica y tecnológica acerca del tratamiento de vertimientos líquidos en industrias de alimentos. Para la realización de la búsqueda de información se consultaron las bases de datos mencionadas en la tabla 1. Teniendo en cuenta que se realizó una búsqueda de información en el ámbito tecnológico, investigativo y comercia, los idiomas para tener en cuenta fueron inglés y español.

Tabla 1. Bases de datos utilizadas para la vigilancia tecnológica.

\begin{tabular}{|l|l|}
\hline \multicolumn{1}{|c|}{ Tipo de información } & \multicolumn{1}{|c|}{ Bases de datos } \\
\hline Científica & $\begin{array}{l}\text { Science direct, Scopus (análisis cienciométrico), } \\
\text { Google académico }\end{array}$ \\
\hline Tecnológica & $\begin{array}{l}\text { Organización mundial de propiedad intelectual } \\
\text { (búsqueda de patentes y análisis cienciométrico), Google } \\
\text { patents }\end{array}$ \\
\hline Comercial & Motor de búsqueda Google \\
\hline
\end{tabular}

\subsection{Palabras claves:}

Liquid, waste, food, industry

\subsection{Ecuación de búsqueda:}

Liquid waste AND food industry AND (2010 OR 2011 OR 2012 OR 2013 OR 2014 OR 2015 OR 2016 OR 2017 OR 2018). 


\section{RESULTADOS}

\subsection{Contextualización de la problemática}

La generación de subproductos alimentarios es un tema que ha sido de interés mundial. La Organización de las Naciones Unidas para la Alimentación y la Agricultura (FAO) ha realizado estudios donde analiza y recopila información acerca del desperdicio de alimentos y su repercusión ambiental y económica, los resultados más relevantes se presentan a continuación.

\subsubsection{Relación de producción de $\mathrm{CO}_{2}$ y generación de subproductos alimentarios}

La inadecuada disposición de subproductos alimenticios líquidos y sólidos (vertimiento a fuentes hídricas o disposición a campo abierto) genera un proceso de degradación que implica la generación de gases del efecto invernadero, entre ellos $\mathrm{CO}_{2}$. En la imagen 1 se relaciona la cantidad de gases de efecto invernadero generados por país versus la cantidad de $\mathrm{CO}_{2}$ generada referente a residuos alimenticios.

\section{Cantidad de $\mathrm{CO} 2$ emitido por país versus generación de $\mathrm{CO} 2$ por pérdidas de alimentos}

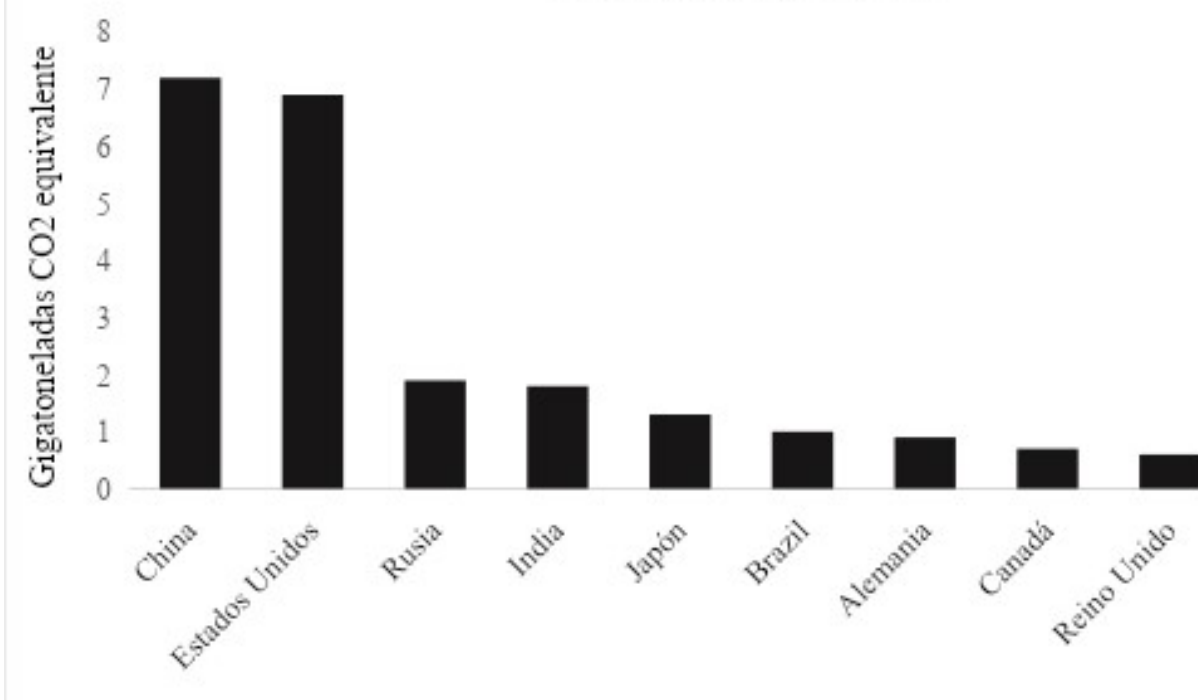

Figura 1. Países que más emiten gases de efecto invernadero a nivel mundial frente a generación de $\mathrm{CO}_{2}$ por pérdida y desperdicio de alimentos. (Adaptado de [9])

\subsubsection{Pérdida de alimentos per cápita}

La figura 2 muestra relaciones entre producción de alimentos según continentes y regiones. Los continentes con mayor cantidad de subproductos en cuanto a consumo son los que presentan mayor desarrollo, mientras que los continentes o regiones en vías de desarrollo muestran mayor desperdicio en su producción, quizás por la poca optimización o tecnificación de los procesos. 


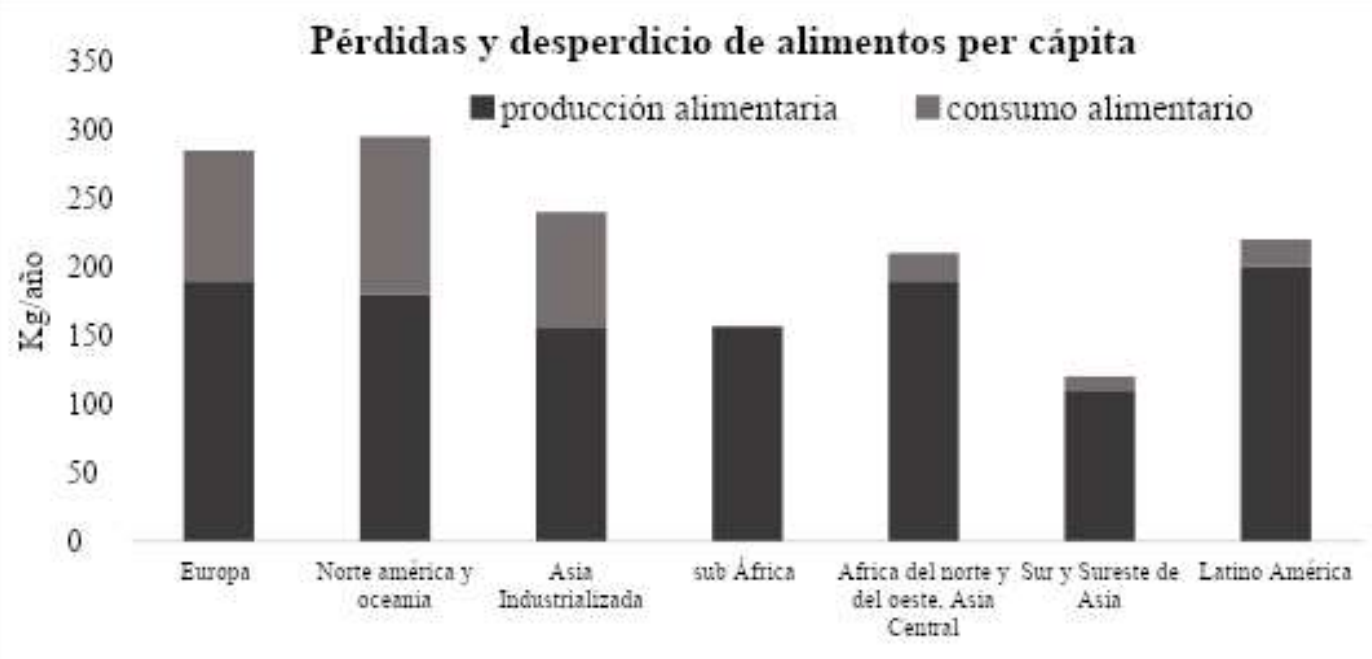

Figura 2. Pérdidas y desperdicio de alimentos per cápita por continentes y regiones. (Adaptado de [10])

Los residuos líquidos varían según el sector industrial o el producto final que se realice [3], entre ellos se pueden encontrar subproductos líquidos con alta cantidad de sólidos suspendidos, grasas, azúcares o almidón. Teniendo en cuenta que, según la composición de los residuos se decide su su tratamiento, resulta de vital importancia conocer de qué están hechos. La tabla 2 resume la composición típica de los residuos alimenticios según su origen.

Tabla 2. Composición típica de los residuos alimenticios. (Adaptado de [3])

\begin{tabular}{|l|l|}
\hline Tipo de residuo & \multicolumn{1}{c|}{ Composición } \\
\hline Carnes/aves & Alto contenido de lípidos emulsificados y proteína. \\
\hline Láctea & Lípidos no emulsificados, lactosa y caseína. \\
\hline Vegetales/frutas & Ricos en azúcares, almidones (carbohidrato y celulosa). \\
\hline Cervecera/licores & Alto contenido de sólidos, proteínas y azúcares fermentables. \\
\hline
\end{tabular}

\subsection{Producción científica}

Se encontraron artículos en 3 grandes campos: aprovechamiento biotecnológico para obtención de productos de valor agregado, tratamiento con enfoque ambiental para mitigación del impacto ambiental (disminución de la carga orgánica) y alimentación animal. La tabla 3 y la Figura 3 resumen la información encontrada.

\section{Tendencia cantidad de artículos por año según área}

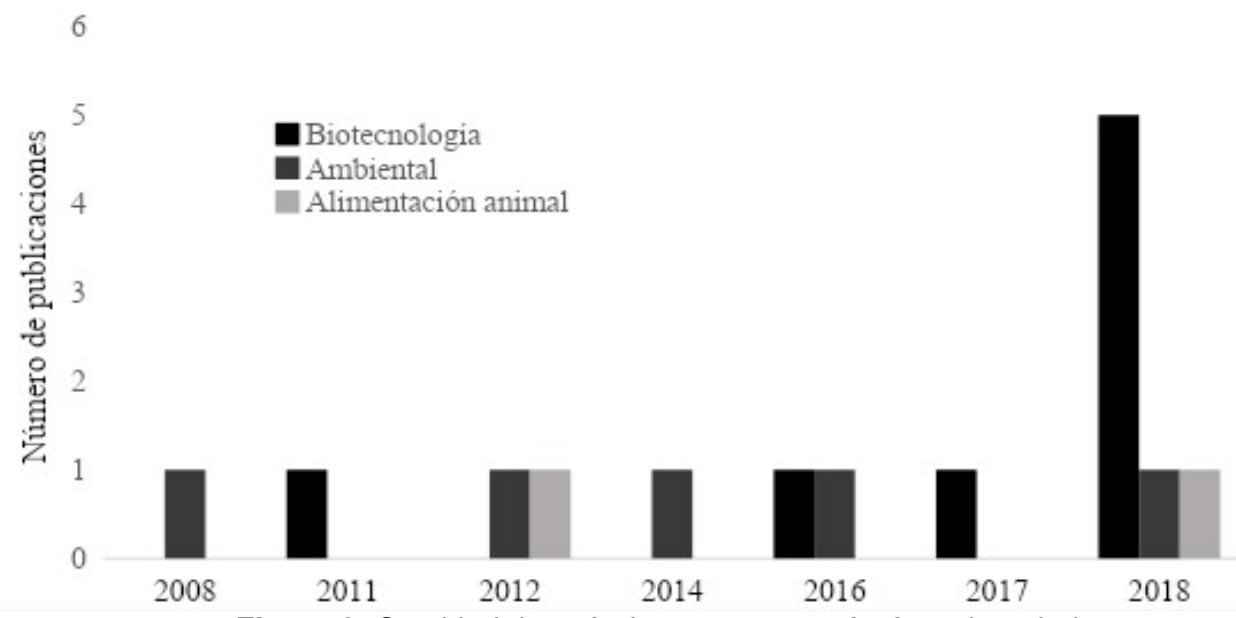

Figura 3. Cantidad de artículos por año según área de trabajo. 


\subsubsection{Tratamiento con enfoque biotecnológico}

Teniendo en cuenta que los subproductos contienen gran cantidad de compuestos que pueden ser de interés, el enfoque biotecnológico trata sobre su conversión en productos de valor agregado o utilización para procesos industriales, promoviendo de esta manera la economía circular y generando beneficios en el ámbito económico.

Tabla 3. Enfoques de aprovechamiento de subproductos líquidos provenientes de industrias de alimentos (FUENTE: elaboración propia).

\begin{tabular}{|c|c|}
\hline Área & Usos \\
\hline $\begin{array}{l}\text { Aprovechamiento } \\
\text { biotecnológico }\end{array}$ & $\begin{array}{l}\checkmark \text { Producción de materias primas almidonadas o con gran } \\
\text { concentración de azúcares por medio de enzimas [11]. } \\
\checkmark \text { Producción de alcoholes por medio de fermentación de } \\
\text { materias primas [12]. } \\
\checkmark \text { Producción de enzimas [13]. } \\
\checkmark \text { Producción de biopolímeros: (polihidroxialcanoatos (PHA), } \\
\text { polihidroxibutiratos (PHB) [13]. } \\
\checkmark \text { Biocombustibles, bioplásticos, solventes [14]. } \\
\checkmark \text { Producción de ácidos grasos con aplicaciones cosméticas, } \\
\text { farmacéuticas y alimenticias [15]. } \\
\checkmark \text { Producción de bebidas alcohólicas a partir de residuos de } \\
\text { frutas [16]. } \\
\checkmark \text { Producción de ésteres y ceras [38]. } \\
\checkmark \text { Digestión anaerobia (producción de gas y energía) [18] [19] } \\
\text { [20][21]. }\end{array}$ \\
\hline $\begin{array}{c}\text { Tratamiento con } \\
\text { enfoque ambiental }\end{array}$ & $\begin{array}{ll}\checkmark & \text { Compostaje [18]. } \\
\checkmark & \text { Evaporación [19]. } \\
\checkmark & \text { Osmosis reversa [20]. } \\
\checkmark & \text { Incineración [21]. } \\
\checkmark & \text { Ozonificación [21]. } \\
\checkmark & \text { Coagulación floculación [22]. } \\
\checkmark & \text { Filtración [20]. } \\
\checkmark & \text { Oxidación electroquímica [21]. } \\
\checkmark & \text { Purificación y obtención de productos (ácidos orgánicos, } \\
& \text { aceites) [22]. }\end{array}$ \\
\hline Alimentación animal & $\checkmark$ Valorización como alimento animal [16], [22]. \\
\hline
\end{tabular}

Un componente de gran importancia para la reutilización de los subproductos son las enzimas, catalizadores biológicos encargados de romper las macromoléculas presentes aún en los subproductos. Enzimas como amilasas y celulasas pueden ser utilizadas en los procesos para producción de biocombustibles, materia prima con alto contenido de azúcares o en la producción de biopolímeros [1]; adicionalmente, es posible obtener grandes cantidades de enzimas usando como medio de cultivo un subproducto, disminuyendo de esta manera (al menos parcialmente) el valor de producción de tales biomoléculas [23].

Bioproductos como los polihidroxialcanoatos (PHA), polihidroxibutiratos (PHB), ácido láctico, acético, solventes, etanol y en general productos fermentados pueden ser obtenidos mediante la conversión de los subproductos usando microrganismos entre los que se destacan géneros como Sacharomyces, Pichia y Clostridium [12]. La obtención de los compuestos previamente mencionados por métodos biotecnológicos disminuye la dependencia del petróleo y los recursos naturales para su producción convencional; adicionalmente, al ser el sustrato de un valor mínimo o inexistente, los costos de producción de los biocompuestos (tales como PHA, PHB, ácido láctico, acético, solventes, etanol) disminuyen. Cepas como Cryptococcus curvatus, Rhodosporidium toruloides y Lipomyces starkeyi pueden producir ceras o ácidos grasos de interés cosmético, farmacéutico y alimenticio a partir de subproductos como residuos de vino y queso [9], eliminando así la dependencia de cultivos y las afectaciones en su producción por las variaciones climáticas existentes [22] [24].

Polímeros como celulosa, quitosano o almidón pueden ser encontrados en subproductos industriales pues son componentes fundamentales de los alimentos. Las moléculas mencionadas pueden ser materia prima en el área 
de nanotecnología, como materia prima para la síntesis de nanopartículas de platino, níquel y plata, algunas de éstas con actividad antibacteriana [13].

Finalmente, una de las metodologías más relevantes del tratamiento de subproductos con enfoque biotecnológico es mediante la producción de energía en dispositivos denominados biodigestores, donde se da la conversión de lodos o material sólido en energía (en forma de calor y gas) [20]. Asimismo, los lodos residuales obtenidos pueden ser utilizados como biofertilizantes [19].

\subsubsection{Tratamiento con enfoque ambiental}

El tratamiento con enfoque ambiental comprende varios procedimientos físicos y químicos. El fin de los procedimientos físicos es la eliminación o reducción del material particulado de gran tamaño, generando como producto final un producto líquido, o con menor cantidad de sólidos [25]. Dentro de las metodologías usadas para tal fin se destacan la ósmosis reversa (mediante un sistema de filtros se retiene el material particulado y algunas moléculas); floculación y coagulación (tratamientos en los cuales se adicionan sustancias químicas que a cierto $\mathrm{pH}$ sedimentan una alta cantidad de sólidos) y finalmente, flotación (tratamiento efectivo para eliminar parcialmente sustancias como grasas) 19]. Las tecnologías previamente mencionadas han sido reportadas para procesos como la remoción de color de aguas residuales [26] y en programas de acueductos urbanos [27], demostrando que son metodologías versátiles.

Posterior a la eliminación del material sólido se ha reportado el uso de evaporación mediante el cual se elimina el alto porcentaje de agua presente en los subproductos líquidos. El remanente de tal proceso puede ser utilizado como fertilizante, esto por su concentración de nitrógeno [26].

Los tratamientos químicos se enfocan en disminuir especies químicas que no son tratables por métodos biológicos o que su conversión afecta el metabolismo celular [28]. Dentro de los tratamientos químicos se encuentra la hidrólisis química, mediante la cual los subproductos son sometidos a una hidrólisis ácida o alcalina a condiciones moderadas de temperatura (entre 60 y $100^{\circ} \mathrm{C}$ ), el producto de tal proceso depende de la materia prima de la cual se partió pero puede estar compuesta de polisacáridos solubles, indicando que tal hidrólisis rompe las cadenas de grandes estructuras generando compuestos de tamaño intermedio como producto final, siendo de esta manera útil como biofertilizante [18]. Otros métodos utilizados son ozonización/UV, degradación aeróbica por medio de biorreactores (entre ellos de membrana), digestión termofílica, incineración, electrodiálisis, pirólisis, compostaje, y oxidación húmeda [37]. Para la elección de la metodología más adecuada para cada caso, es relevante analizar costos, infraestructura necesaria y eficiencia del proceso [21].

Uno de los residuos más relevantes en las plantas de sacrificio y el consumo avícola es la sangre, rica en materia orgánica y baja en carbohidratos. A partir de este subproducto pueden obtenerse compuestos como albúmina, globulina y fibrinógeno que pueden ser usados para la elaboración de proteínas hiladas, con características similares a las encontradas en la fibra de la carne [27].

Finalmente, en el libro "Waste Management for the Food Industries" se mencionan varios métodos de tratamiento de residuos de alimentos según su procedencia (cerveza, vino, procesos de destilación, bebidas, frutas y vegetales, carnes y residuos agroindustriales); la tabla 4 resume los hallazgos más relevantes asociados a residuos líquidos generados en diversas industrias [21].

Tabla 4. Metodologías de tratamiento de subproductos en la producción de diversos alimentos (Adaptado de [21]).

\begin{tabular}{|c|c|}
\hline Proceso & Tratamiento \\
\hline $\begin{array}{l}\text { Producción de vinos: aguas del tratamiento de } \\
\text { zumo de uvas }\end{array}$ & Electrodiálisis. \\
\hline $\begin{array}{l}\text { Procesamiento de oliva: residuos de } \\
\text { producción de aceite de oliva }\end{array}$ & $\begin{array}{l}\text { Biorremediación, procesos térmicos, filtración } \\
\text { por membrana, electrólisis, ozonización, } \\
\text { digestión, coagulación/filtración, destilación. }\end{array}$ \\
\hline $\begin{array}{l}\text { Producción de jugos y pulpas: lixiviados de } \\
\text { producción. }\end{array}$ & Ósmosis reversa, evaporación. \\
\hline $\begin{array}{l}\text { Producción de aceite de palma: Efluente } \\
\text { molido aceite de palma }\end{array}$ & Ultrafiltración. \\
\hline Procesamiento del maíz: aceite de maíz & Fermentación. \\
\hline $\begin{array}{l}\text { Procesamiento de lácteos: residuos de la } \\
\text { industria láctea }\end{array}$ & $\begin{array}{l}\text { Digestión anaeróbica, nanofiltración, ósmosis } \\
\text { reversa, filtración por membrana, coagulación, } \\
\text { oxidación electroquímica, coagulación. }\end{array}$ \\
\hline Procesamiento de carnes: caso pescado. & $\begin{array}{l}\text { Digestión anaeróbica, filtración, evaporación, } \\
\text { coagulación, floculación. }\end{array}$ \\
\hline
\end{tabular}




\subsubsection{Tratamiento con enfoque ambiental}

Bajo los mismos criterios de búsqueda de la información científica se realizó la búsqueda de carácter tecnológico. La base de datos WIPO arrojó en total 19 reportes en el intervalo de tiempo evaluado. En la figura 4 se relaciona la producción tecnológica de los últimos 10 años, donde se puede observar que la temática "vertimientos líquidos en industrias de alimentos" tuvo su mayor cantidad de producción tecnológica en el año 2014 y continuó con comportamiento principalmente a la baja en los años siguientes.

\section{Cantidad de patentes por año}

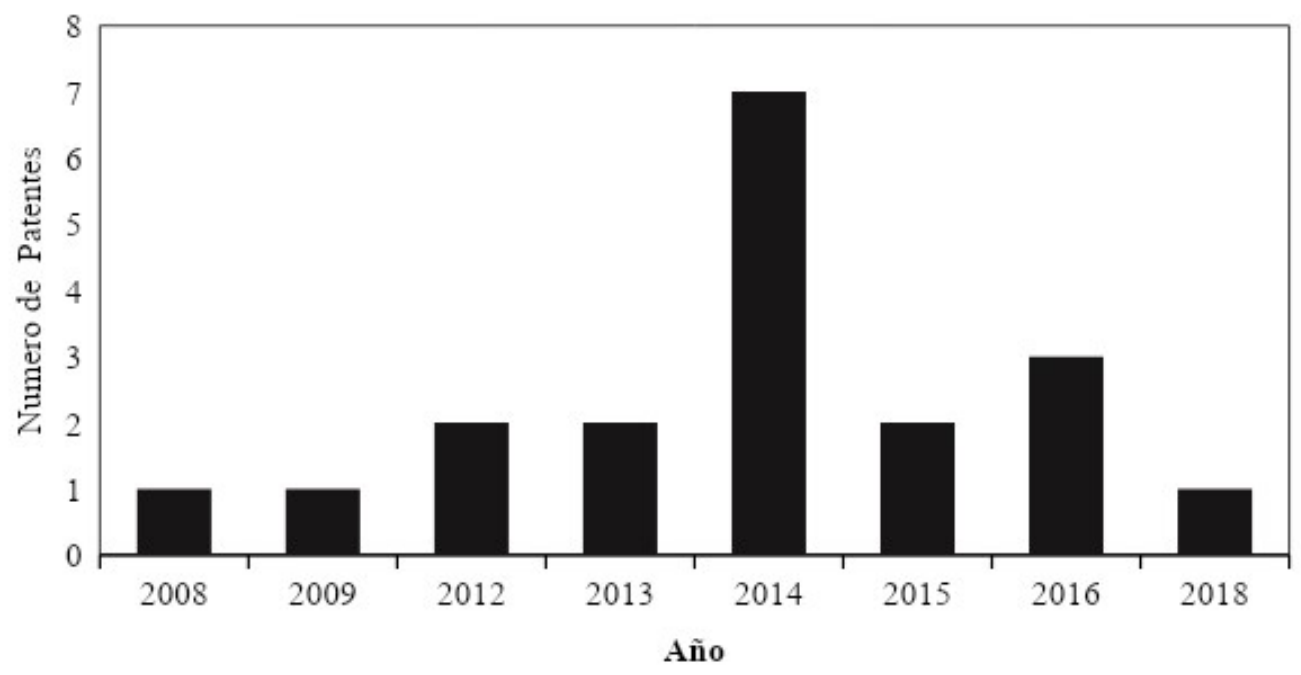

Figura 4. Registro número de patentes entre 2008-2018.

Respecto al área causal de protección, se puede observar relevancia en patentes asociadas a la producción de fertilizantes, fermentaciones y el diseño de dispositivos para el tratamiento de subproductos específicos, siendo entonces una temática más enfocada a metodologías de obtención o preparación de compuestos que al diseño de equipos para el área. La tabla 5 resume la información encontrada.

Tabla 5. Clasificación de patentes encontradas para el objeto de estudio: "vertimientos líquidos en industrias de alimentos".

\begin{tabular}{|l|l|}
\hline Código & Descripción \\
\hline C05F & Desarrollo de fertilizantes orgánicos \\
\hline C02F & Tratamiento de aguas mediante reacciones químicas \\
\hline C05D & Fertilizantes con potasio \\
\hline C12P & Procedimientos que involucran fermentación \\
\hline B01F & Dispositivos para dispersar o emulsificar. \\
\hline C05B & Fertilizantes hechos a base de reacciones químicas \\
\hline C05C & Fertilizantes a base de nitratos \\
\hline C09B & Fermentación para obtener un compuesto deseado \\
\hline C11B & Obtención de grasas, aceites y otras materias primas \\
\hline C12N & Bioformulados \\
\hline
\end{tabular}

Respecto a las empresas que más generan protección de tecnología para el tratamiento de subproductos líquidos en industrias de alimentos, se encuentra que la organización MANETECH (8 reportes) es una empresa dedicada a resolver problemas ambientales, con sede en República Checa. En segundo lugar, se destaca River Road Research (6 reportes), empresa dedicada al desarrollo de tecnologías que permiten la eliminación completa de los residuos de alimentos de una manera económica y ecológicamente positiva. La empresa es estadounidense y tiene sede en California. La Figura 5 relaciona las entidades que han patentado en el área. En la tabla 6 se resumen las patentes más relevantes en el área de vertimientos líquidos en industrias de alimentos. 
Avances tecnológicos en gestión de vertimientos líquidos en plantas de producción de alimentos Technological advances in liquid watershed management from food production centers

Tabla 6. Resumen de las patentes más relevantes en el área.

\begin{tabular}{|c|c|c|c|c|}
\hline Registro & Año & Especificaciones & $\begin{array}{cccc}\begin{array}{c}\text { Imagen } \\
\text { patentada }\end{array} & \text { de la } & & \text { tecnología } \\
\end{array}$ & Referencia \\
\hline CN103868076A & 2014 & $\begin{array}{l}\text { Equipo } \text { incinerador de } \\
\text { desechos líquidos y sólidos, } \\
\text { incluye cámara de combustión, } \\
\text { sistema de secado por } \\
\text { aspersión, una cámara } \\
\text { secundaria de secado }\end{array}$ & c. & [29] \\
\hline WO2018032119A1 & 2018 & $\begin{array}{l}\text { Equipo para tratamiento de } \\
\text { residuos industriales líquidos, } \\
\text { portátil y con varias unidades } \\
\text { independientes para filtrar, } \\
\text { controlar olores y digerir } \\
\text { desechos. }\end{array}$ & 8080800 & [30] \\
\hline CN203582529U & 2013 & $\begin{array}{l}\text { La patente } \\
\text { sistema de evaporaciona un } \\
\text { cristalización y de aguas } \\
\text { residuales orgánicas, comprende } \\
\text { un tanque de evaporación del } \\
\text { líquido, un compresor y un } \\
\text { intercambiador de calor. }\end{array}$ & & [31] \\
\hline CN203159266U & 2013 & $\begin{array}{l}\text { El dispositivo consta con un } \\
\text { sinnúmero de barras, dentro del } \\
\text { sistema los residuos se } \\
\text { calientan. Se menciona que el } \\
\text { dispositivo es altamente eficiente } \\
\text { y tiene un bajo costo de } \\
\text { inversión. }\end{array}$ & $\stackrel{B}{-}$ & [32] \\
\hline & & & As & \\
\hline WO2015137966A1 & 2014 & $\begin{array}{l}\text { La invención menciona el uso } \\
\text { de compuestos químicos tipo } \\
\text { inulina y derivados, éstos tienen } \\
\text { una modificación con un sitio de } \\
\text { carga catiónica para mejorar la } \\
\text { unión del material con el residuo. }\end{array}$ & $\mathrm{N} / \mathrm{A}$ & [33] \\
\hline RU02320547 & 2008 & $\begin{array}{l}\text { Se propone } \\
\text { aprovechamiento de residuos de } \\
\text { procesos de sustratos } \\
\text { almidonados, tipo granos y papa. } \\
\text { El proceso inicia con la remoción } \\
\text { de material particulado, las } \\
\text { aguas remanentes pasan a un } \\
\text { proceso de aireación y de } \\
\text { remoción de carga orgánica } \\
\text { mediante el uso de lodos } \\
\text { activados, en varios tanques. }\end{array}$ & $\mathrm{N} / \mathrm{A}$ & [34] \\
\hline US20160083309A1 & 2014 & $\begin{array}{l}\text { Utilización de un biorreactor } \\
\text { para procesamiento de } \\
\text { subproductos }\end{array}$ & N/A & [35] \\
\hline RU2531931C1 & 2013 & \begin{tabular}{l}
\multicolumn{1}{c}{ Tratamiento de aguas } \\
residuales con un coagulante \\
compuesto \\
provenientes de le lodos aguas \\
residuales, disminuyendo la \\
carga orgánica en efluentes de \\
industrias de alimentos y \\
recuperando componentes \\
valiosos para el aumento de los \\
lodos, facilitando su cultivo.
\end{tabular} & Фиr.1 & {$[36]$} \\
\hline
\end{tabular}




\section{Organizaciones que patentan en el área de vertimientos \\ líquidos en industrias de alimentos}

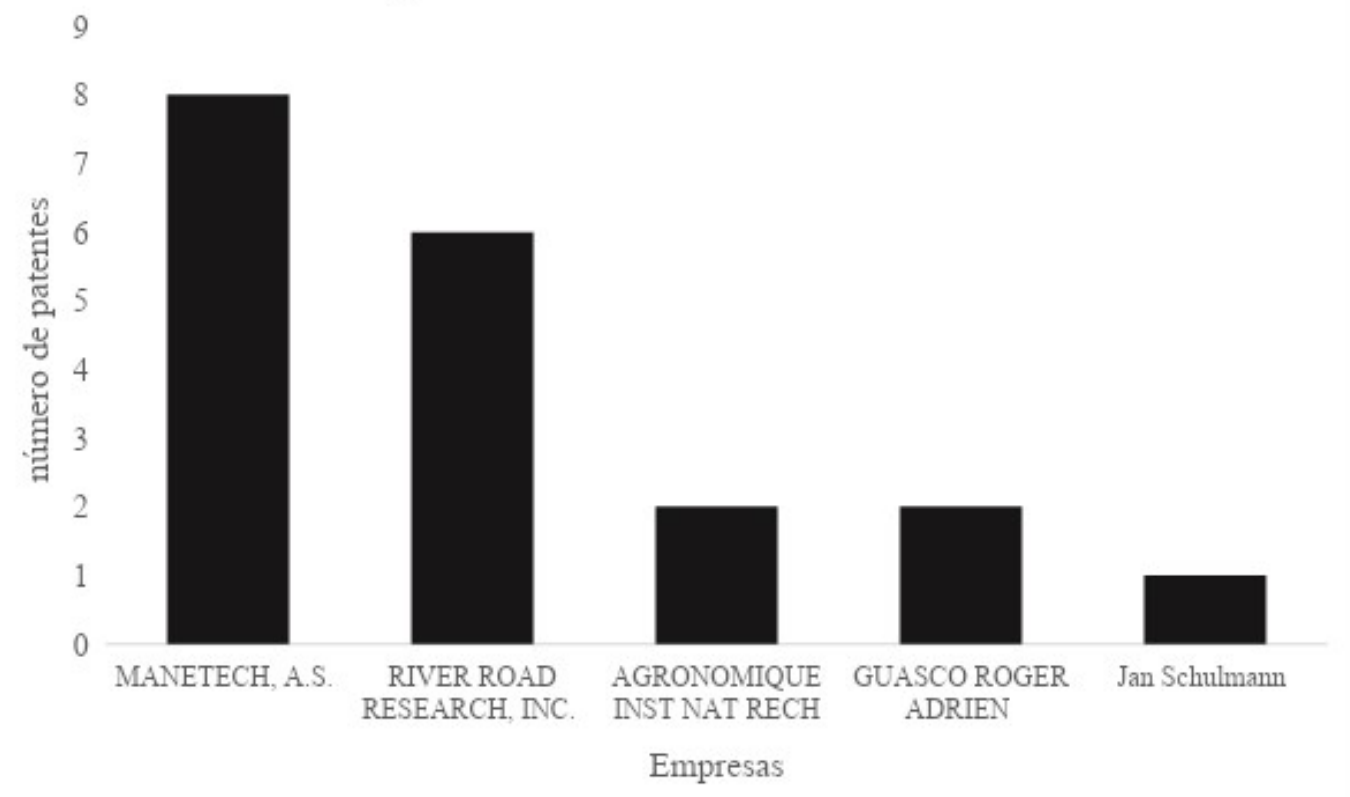

Figura 5. Relación de entidades que han patentado en el tema vertimientos líquidos en industrias de alimentos.

\subsection{Búsqueda de información de carácter comercial}

Finalmente, se realizó una búsqueda de información de carácter comercial encontrando los proveedores que se listan en la tabla 7, siendo en su mayoría empresas fuera de Colombia.

\section{CONCLUSIONES Y TRABAJOS FUTUROS}

Actualmente, uno de los principales aspectos que suscita el interés general de las autoridades ambientales y empresas a nivel mundial, radica en la prevención de la contaminación ambiental generada por los subproductos líquidos, disponiendo éstos de otra manera, reutilizándolos y generando ingresos adicionales a partir de la obtención de nuevos productos. Este trabajo presentó una búsqueda, análisis y recopilación de información de carácter científico, tecnológico y comercial en el tema de vertimientos líquidos en industrias de alimentos.

Existen diversas metodologías para el tratamiento de residuos líquidos. Es relevante hacer una caracterización del subproducto generado para así conocer las posibilidades de uso y enfocar la producción de un compuesto o grupo de compuestos.

La temática aquí tratada ha sido abarcada especialmente desde el ámbito investigativo. Se encontraron pocas empresas que se dediquen a dar solución a los vertimientos líquidos en industrias de alimentos; de la misma manera, la cantidad de patentes encontrada no fue alta, encontrando mayor cantidad de información tecnológica en el área de procedimientos o protocolos para la obtención de abonos orgánicos. Los resultados indican que el proceso más usado para el tratamiento de residuos líquidos es mediante su deshidratación y tratamiento en plantas de aguas residuales; sin embargo, debido a su composición, es relevante implementar economía circular y usar dichos subproductos como materia prima para la producción de jarabes con alto contenido de azúcar, producción de alcoholes, enzimas, biopolímeros, entre otros. El análisis cienciométrico indica que España y Estados Unidos son pioneros en las investigaciones y patentes en el tema, mientras Colombia no presenta publicaciones entre 2008 y 2018. 
Tabla 7. Información comercial acerca de tratamiento para vertimientos líquidos en industrias de alimentos.

\begin{tabular}{|c|c|c|c|}
\hline Empresa & País & Productos & Enlace página web \\
\hline $\begin{array}{l}\text { Jinp } \\
\text { indu }\end{array}$ & China & $\begin{array}{l}\text { Máquinas para procesamiento de } \\
\text { residuos por medio de pirolisis } \\
\text { (incineración a alta temperatura en } \\
\text { ausencia de oxígeno). Disposición de } \\
\text { residuos domésticos, de llantas o } \\
\text { neumáticos, residuos plásticos, } \\
\text { residuos de aceites. Los equipos son } \\
\text { voluminosos y a escala industrial. }\end{array}$ & $\begin{array}{l}\text { http://www.pyrolysis- } \\
\text { machine.com/index.html }\end{array}$ \\
\hline $\begin{array}{l}\text { SSI } \because: \because{ }^{\circ} \\
\text { SSI } \\
\text { Aeration }\end{array}$ & EE. UU. & $\begin{array}{l}\text { Plantas de tratamiento de aguas } \\
\text { residuales. Cuentan con experiencia } \\
\text { en: pulpa, papel, productos lácteos, } \\
\text { procesamiento de carne, curtimbres, } \\
\text { bebidas. }\end{array}$ & $\begin{array}{l}\text { https://www.ssiaeratio } \\
\text { n.com/industrial/ }\end{array}$ \\
\hline Soclean & India & $\begin{array}{l}\text { Equipos de tratamientos de residuos } \\
\text { líquidos, los equipos son más } \\
\text { pequeños ( } 75-100 \text { litros) }\end{array}$ & / https://www.soclean.in \\
\hline & Japón & $\begin{array}{l}\text { Bajo costo inicial, bajo costo de } \\
\text { funcionamiento, pudiéndose aplicar a } \\
\text { sistemas compactos y grandes } \\
\text { instalaciones según su naturaleza, } \\
\text { calidad del agua, caudal etc. }\end{array}$ & $\begin{array}{l}\text { https://www.sinto.co.jp } \\
\text { /en/product/environment/ } \\
\text { water/index.html }\end{array}$ \\
\hline M & China & $\begin{array}{l}\text { Equipos de tratamiento de aguas } \\
\text { residuales (según su tipo). Ofrecen } \\
\text { sistemas de tratamiento de aguas } \\
\text { residuales estables para aguas turbias, } \\
\text { aguas residuales orgánicas, aguas } \\
\text { residuales que contienen petróleo y } \\
\text { aguas residuales de medicamentos/ } \\
\text { revestimientos, recubrimientos y } \\
\text { recubrimientos en diversas áreas y } \\
\text { campos industriales. }\end{array}$ & $\begin{array}{l}\text { http://www.yzmaoyuan } \\
\text {.com/en/a/guanyuwomen } \\
\text { / }\end{array}$ \\
\hline $\begin{array}{l}\text { Cor } \\
\text { m env }\end{array}$ & $\begin{array}{l}\text { Europa, } \\
\text { EE. UU., } \\
\text { México }\end{array}$ & $\begin{array}{l}\text { Técnicas: evaporación al vacío } \\
\text { (cristalizadores térmicos). Tecnologías } \\
\text { de baja potencia y bajo costo de } \\
\text { administración }\end{array}$ & $\begin{array}{l}\text { https://condorchem.co } \\
\text { m/es/ }\end{array}$ \\
\hline Nyf sinergy & ia & $\begin{array}{l}\text { Compañía con más de } 29 \text { años de } \\
\text { experiencia, dedicada a la fabricación e } \\
\text { implementación de sistemas para } \\
\text { tratamiento de aguas, con la finalidad } \\
\text { de tratar y recuperar el agua de } \\
\text { cualquier tipo, obteniendo con esto que } \\
\text { las fuentes de agua no sean sobre } \\
\text { explotadas logrando la recuperación } \\
\text { natural de las mismas }\end{array}$ & $\begin{array}{l}\text { https://www.nyfdecolo } \\
\text { mbia.com/ }\end{array}$ \\
\hline
\end{tabular}




\section{REFERENCIAS}

[1] MINISTRY OF ENVIRONMENT AND WATER, "NATIONAL WASTE MANAGEMENT PLAN 2014-2020," Sofia, Bulgaria, 2014.

[2] J. Gustavsson, C. Cederberg, U. Sonesson, R. Van Otterdijk, A. Meybeck, and F. Rome, "FAO - News Article: Cutting food waste to feed the world," 2011.

[3] F. M. Castro Aravena, "Estudio de los residuos industriales líquidos y evaluación de las alternativas de mejora en la industria cervecera Valdivia," Universidad Austral de Chile, 2003.

[4] European Commission European, “PREPARATORY STUDY ON FOOD WASTE ACROSS EU 27," 2010. [5] Food processing industry news, "What a waste of potatoes," 2015. [Online]. Available: https://www.foodprocessing.com.au/content/sustainability/news/what-a-waste-of-potatoes-445666004.

[Accessed: 07-Dec-2019].

[6] R. Devesa-Rey, X. Vecino, J. L. Varela-Alende, M. T. Barral, J. M. Cruz, and A. B. Moldes, "Valorization of winery waste vs. the costs of not recycling," Waste Manag., vol. 31, no. 11, pp. 2327-2335, Nov. 2011.

[7] Y. Zhang, X. C. Wang, Z. Cheng, Y. Li, and J. Tang, "Effect of fermentation liquid from food waste as a carbon source for enhancing denitrification in wastewater treatment," Chemosphere, vol. 144, pp. 689-696, Feb. 2016.

[8] D. M. Gómez Quintero and A. I. González Alcalá, "Il Guía práctica Innovitech: vigilancia tecnológica para la innovación," Servicio Nacional de Aprendizaje (SENA), 2016.

[9] (FAO) Food wastage footprint, "Food Wastage Footprint - Impacts on Natural Resources - Summary report," 2013.

[10] (FAO) Food wastage footprint, "Global Food Losses and Food Waste: Extent, Causes and Prevention World | ReliefWeb," 2011.

[11] T. H. Kwan, K. L. Ong, M. A. Haque, W. H. Kwan, S. Kulkarni, and C. S. K. Lin, "Valorisation of food and beverage waste via saccharification for sugars recovery," Bioresour. Technol., vol. 255, pp. 67-75, May 2018.

[12] S. Hegde, J. S. Lodge, and T. A. Trabold, "Characteristics of food processing wastes and their use in sustainable alcohol production," Renewable and Sustainable Energy Reviews, vol. 81. Elsevier Ltd, pp. 510-523, 2018.

[13] R. Ravindran and A. K. Jaiswal, "Exploitation of Food Industry Waste for High-Value Products," Trends in Biotechnology, vol. 34, no. 1. Elsevier Ltd, pp. 58-69, 01-Jan-2016.

[14] M. Esteban-Gutiérrez, J. Garcia-Aguirre, I. Irizar, and E. Aymerich, "From sewage sludge and agri-food waste to VFA: Individual acid production potential and up-scaling," Waste Manag., vol. 77, pp. 203-212, Jul. 2018.

[15] A. Monteiro et al., "Liquid by-products from fish canning industry as sustainable sources of $\omega 3$ lipids," $J$. Environ. Manage., vol. 219, pp. 9-17, Aug. 2018.

[16] M. G. Cruz et al., "Waste mitigation: From an effluent of apple juice concentrate industry to a valuable ingredient for food and feed applications," J. Clean. Prod., vol. 193, pp. 652-660, Aug. 2018.

[17] O. Guneser, A. Demirkol, Y. Karagul Yuceer, O. Togay, I. Hosoglu, and M. Elibol, "Biotechnology and Industrial Microbiology Production of flavor compounds from olive mill waste by Rhizopus oryzae and Candida tropicalis," Brazilian J. Microbiol., vol. 48, pp. 275-285, 2017.

[18] C. Du et al., "Valorization of food waste into biofertiliser and its field application," J. Clean. Prod., vol. 187, pp. 273-284, Jun. 2018.

[19] P. Zhang, C. J. Lin, J. Liu, P. Pongprueksa, S. A. Evers, and P. Hart, "Biogas production from brown grease using a pilot-scale high-rate anaerobic digester," Renew. Energy, vol. 68, pp. 304-313, Aug. 2014.

[20] L. Guerrero Saldes and D. Alkalay Lowitt, "TRATAMIENTO DE RESIDUOS LÍQUIDOS DE UNA FÁBRICA DE GALLETAS."

[21] I. S. Arvanitoyannis, Waste Management for the Food Industries, 16th ed. Elsevier Inc., 2008.

[22] K. Rezzadori, S. Benedetti, and E. R. Amante, "Proposals for the residues recovery: Orange waste as raw material for new products," Food and Bioproducts Processing, vol. 90, no. 4. pp. 606-614, Oct-2012.

[23] D. Klein-Marcuschamer, P. Oleskowicz-Popiel, B. A. Simmons, and H. W. Blanch, "The challenge of enzyme cost in the production of lignocellulosic biofuels," Biotechnol. Bioeng., vol. 109, no. 4, pp. 1083-1087, Apr. 2012.

[24] E. C. G. Aguieiras, E. D. Cavalcanti-Oliveira, and D. M. G. Freire, "Current status and new developments of biodiesel production using fungal lipases," Fuel, vol. 159, pp. xiii-xv, 2015.

[25] E. Mejía, L. Osorno, and J. Ospina, "Microorganismos Hierro-Azufre Oxidantes Una Alternativa Biotecnológica," Rev. CINTEX, vol. 19, pp. 63-77, 2014

[26] G. D. Jojoa-Unigarro, H. L. Rodríguez-Zambrano, and S. A. Cardona-Gallo, "Tratamiento de aguas residuales textiles a partir de métodos biológicos," Rev. CINTEX, vol. 20, no. 1, pp. 11-34, jun. 2015. 
[27] S. Gabarrón, M. Dalmau, H. Monclús, J. Comas, and I. Rodriguez-Roda, "Design and operational issues of 7 full-scale MBRs for municipal wastewater treatment," Rev. CINTEX, vol. 20, no. 1, pp. 35-51, 2015.

[28] R. H. YOUNG and R. A. LAWRIE, "Utilization of edible protein from meat industry by-products and waste," Int. J. Food Sci. Technol., vol. 9, no. 2, pp. 171-177, Jun. 2007.

[29] Z. C. Y. Chuan, H. Chengguo, T. Yilei, C. Hongyang, G. Zheng, Z. Heliu, Z. Bo, W. Wei, L. Pengfei, J. Fan, Z. Xiaonan, X. Yang, Y. Guohua, "RU2531931C1 - Method of physical and chemical wastewater treatment Google Patents," 2014.

[30] M. A. Molina Ranaval, "SISTEMA DE TRATAMIENTO DE RESIDUOS LÍQUIDOS MODULAR MÓVIL CAMPO DE LA INVENCIÓN,” 2016.

[31] Z. X. J. Lina, "CN203582529U - Evaporation crystallization system for processing saline organic wastewater/liquid waste - Google Patents," 2013.

[32] Q. Pansheng, "CN203159266U - Integrated liquid waste and sludge treatment device - Google Patents," 2013.

[33] S. R. Temple and H. Everett, "WO2015137966A1 - Methods for treating liquid streams containing quaternary ammonium compounds - Google Patents," 2014.

[34] S. Viktorija Nikolaevna, "RU02320547 WASTEWATER TREATMENT PROCESS," 2008.

[35] T. Pinia, "US20160083309A1 - System, method, and composition for enhancing solutions from bioreactors for processes including liquid fertilizer preparation and nutrient extraction - Google Patents," 2016.

[36] U. V. Z. Vasilyeva, Y. Barasheya, "RU2531931C1: Method of physical and chemical wastewater treatment," 2013.

[37] J. L. Palacio, M. Rincón Fulla, and I. Rivera, "Modelo Físico-Matemático para la Estimación del Tamaño de Partículas en Suspensiones Coloidales de Baja Dilución," Rev. CINTEX, vol. 20, no. 1, pp. 53-68, 2015.

[37] A. Papadaki et al., "Production of wax esters via microbial oil synthesis from food industry waste and byproduct streams," Bioresour. Technol., vol. 245, pp. 274-282, 2017 